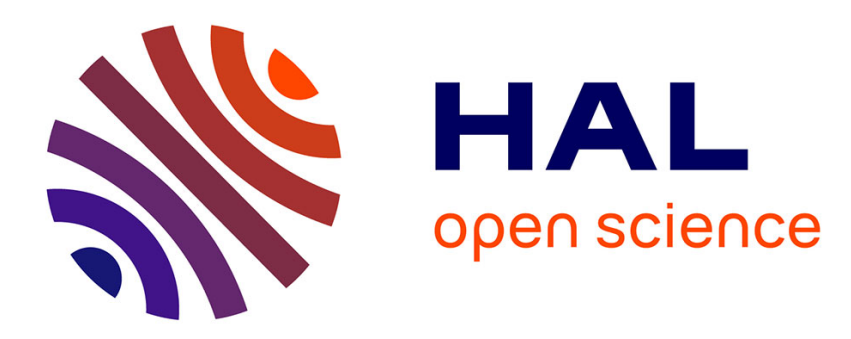

\title{
On functions which are limits of domino tilings \\ Eric Remila
}

\section{To cite this version:}

Eric Remila. On functions which are limits of domino tilings. [Research Report] LIP RR-1999-51, Laboratoire de l'informatique du parallélisme. 1999, 2+13p. hal-02101982

\section{HAL Id: hal-02101982 \\ https://hal-lara.archives-ouvertes.fr/hal-02101982}

Submitted on 17 Apr 2019

HAL is a multi-disciplinary open access archive for the deposit and dissemination of scientific research documents, whether they are published or not. The documents may come from teaching and research institutions in France or abroad, or from public or private research centers.
L'archive ouverte pluridisciplinaire HAL, est destinée au dépôt et à la diffusion de documents scientifiques de niveau recherche, publiés ou non, émanant des établissements d'enseignement et de recherche français ou étrangers, des laboratoires publics ou privés. 
Laboratoire de l'Informatique du Parallélisme

École Normale Supérieure de Lyon

Unité Mixte de Recherche CNRS-INRIA-ENS LYON

$\mathrm{n}^{\mathrm{o}} 5668$



\section{On functions which are limits of domino} tilings

Eric Rémila

november 1999

École Normale Supérieure de Lyon

46 Allée d'Italie, 69364 Lyon Cedex 07, France Téléphone : $+33(0) 4.72 .72 .80 .37$

Télécopieur : $+33(0) 4.72 .72 .80 .80$ Adresse électronique : lip@ens-lyon.fr 


\title{
On functions which are limits of domino tilings
}

\author{
Eric Rémila
}

november 1999

\begin{abstract}
In this paper, we study domino tilings of polygons. We are especially interested in what happens when the domino prototiles become smaller and smaller. This study is done using tiling height functions, which are a numerical way to encode tilings.

The main result of this paper is an analytic characterization of functions which are limits of height functions when the size of dominoes converges to 0 . It is obtained from lattice properties of sets of tilings induced by height functions.
\end{abstract}

Keywords: tiling, height function

\section{Résumé}

nous tudions ici les pavages de polygones par des dominos. Nous nous intressons en particulier ce qui ce passe quand ces dominos deviennent de plus en plus petits. Cette tude est faite au moyen des fonctions de hauteur des pavages, qui permettent de coder les pavages de manire numrique.

Le rsultat principal de ce papier est une caractrisation analytique des fonctions qui se trouvent être des limites des fonctions de hauteur, quand la taille des dominos tend vers 0 . Il est obtenu partir des proprits de treillis des ensembles de pavages, induites par les fonctions de hauteur.

Mots-clés: pavage, fonction de hauteur 


\title{
On functions which are limits of domino tilings
}

\author{
Eric Rémila \\ LIP, umr 5668 CNRS, ENS Lyon \\ 46 allée d'Italie, 69364 Lyon cedex 07, France \\ and \\ GRIMA, IUT Roanne, Université Jean Monnet Saint-Etienne \\ 20 avenue de Paris, 42334 Roanne cedex, France \\ Eric.Remila@univ-st-etienne.fr
}

\begin{abstract}
In this paper, we study domino tilings of polygons. We are especially interested in what happens when the domino prototiles become smaller and smaller. This study is done using tiling height functions, which are a numerical way to encode tilings.

The main result of this paper is an analytic characterization of functions which are limits of height functions when the size of dominoes converges to 0 . It is obtained from lattice properties of sets of tilings induced by height functions.
\end{abstract}

\section{Introduction}

The study of tilings is a classical subject in mathematics. They appear in computer science with the famous result of Berger [1], which proves the indecidability of the problem of knowing if, given a finite set of prototiles, the whole plane can be tiled only using copies of prototiles.

During the last ten years, a lot of advancements have been done about the understanding of tilings. Especially, W. P. Thurston [8] introduced the notion of height function of a tiling with dominoes (i. e. rectangles $2 \times 1$ ), which is a numerical way to encode such a tiling.

From the height functions, W. P. Thurston exhibited a algorithm which, given a polygon $P$ with vertical and horizontal sides, gives a domino tiling of $P$ (or indicates that there is no tiling).

The notion of height function appears as a very powerful tool in tiling study. It has later been adapted by different authors ([3], [5], [7]), who produced tiling algorithms for other sets of prototiles.

In this paper, height functions are used in another direction. Informally, we want to solve the following experiment : take a polygon $P$ : a tiling with dominoes induces a height function $h_{0}$, defined on points of $P$ with integer coordinates. Now, change of scales taking a tiling of $P$ with $p$-dominoes, i. e. $2 / 2^{p} \times 1 / 2^{p}$ rectangles. A tiling of $T$ with $p$-dominoes induces a height function $h_{0}$, defined on points of $P$ of type $\left(a 2^{p}, b 2^{p}\right)$, with $a$ and $b$ integers. 
What happens when $p$ is going to infinity? What are the functions which are "limits of tilings" ? In section 2, we recall definitions and basic properties of tiling height functions, and in order to give a precise formulation of our problem. In section 3, we give some analytic necessary conditions for a function to be a limit of tilings, and in section 4, we prove that each function satisfying these necessary conditions can be approached by a tiling function.

Notice that the main tool for this study is the lattice structure, induced by height functions, of sets of tilings, which is a combinatorial tool, and not an analytic one.

This paper was inspired by a physical analogy. A solid is formed from an arrangement of microscopic atoms, which is a combinatorial structure, but (except if we have complex tools), we can only get macroscopic values (like curvature, density ...) about the solid, which are, in a certain way, limits of the combinatorial structure and have no meaning in a microscopic point of view. Here, we see how the link between macroscopic and microscopic (or discrete and continuous) notions can be done.

The same problem can be easily stated for tilings with calissons (i.e. lozenges an angle of which is $\Pi / 3$ ), and we hope that it can be solved using similar techniques.

\section{Tilings and their height functions}

\subsection{Tilings with $p$-dominoes}

We work in the Euclidean plane $R^{2}$. Let $p$ be a fixed integer. A point $v$ of the plane is a $p$-point (we also say a $p$-vertex) if there exists a pair $(a, b)$ of integers such that : $v=\left(a / 2^{p}, b / 2^{p}\right)$. The set of $p$-points is denoted by $V_{p}$. Notice that if $p<p^{\prime}$, then $V_{p} \subset V_{p^{\prime}}$. Also notice that the set $V=\cup_{p \in Z} V_{p}$ is a dense subset of the plane.

Let $v$ and $v^{\prime}$ be $p$-points. We say that the segment $\left[v, v^{\prime}\right]$ is a $p$-edge and $v^{\prime}$ is a $p$-neighbor of $v$ if the length of $\left[v, v^{\prime}\right]$ is $1 / 2^{p}$. Notice that each $p$-edge is either vertical or horizontal. A $p$-cell is a (closed) square whose sides ares $p$-edges. We divide the set of $p$-cells into black $p$-cells and white $p$-cells according to the lower left corner $v$ of the cell : if $v=\left(a / 2^{p}, b / 2^{p}\right)$, with $a+b$ even, then the cell is white ; otherwise, the cell is black.

This coloration of edges permits to direct $p$-edges : for each pair $\left(v, v^{\prime}\right)$ of $p$-neighbors points, we define the spin of the ordered pair $\left(v, v^{\prime}\right)$ (denoted by $\left.s p\left(v, v^{\prime}\right)\right)$ by : $s p\left(v, v^{\prime}\right)=1$ if $\left(v, v^{\prime}\right)$ if an ant moving from $v$ to $v^{\prime}$ has a white cell on its left side (and a black cell on its right side), and $s p\left(v, v^{\prime}\right)=-1$ otherwise.

A $p$-path is a sequence $\left(v_{0}, v_{1}, \ldots, v_{n}\right)$ of $p$-vertices such that, for each integer $i$ such that $0 \leq i<n, v_{i+1}$ is a $p$-neighbor of $v_{i}$. With these notations we say that $\left(v_{i}, v_{i+1}\right)$ is a $p$-move of the path. We canonically have rightwards, leftwards, upwards and downwards $p$-moves.

The length of such a path is $n / 2^{p}$, and its height difference is the sum $\sum_{i=0}^{n-1} \operatorname{sp}\left(v_{i}, v_{i+1}\right) / 2^{p}$.

A $p$-polygon is a polygon (each side of which is either horizontal or vertical) which is a finite union of $p$-cells. We only consider (which can be done without 
loss of generality, up to translation) polygons a south-west corner of which is the origin $O=(0,0)$. The set of these $p$-polygons is denoted by $S_{p}$. Notice that if $p<p^{\prime}$, then $S_{p} \subset S_{p^{\prime}}$. We also state $S=\cup_{p \in Z} S_{p}$.

A $p$-domino is a rectangle whose dimensions are $1 / 2^{p}$ and $2 / 2^{p}$. A $p$-tiling $T$ of a subset $F$ of the plane is a set of $p$-dominoes with pairwise disjoint interiors whose union equals $F$. A legal path of such a tiling is a $p$-path $\left(v_{0}, v_{1}, \ldots, v_{n}\right)$ such that, for each integer $i$ such that $0 \leq i<n$, the line segment $\left[v_{i}, v_{i+1}\right]$ is included in the boundary of a $p$-domino of $T$.

We say that a polygon $P$ can be $p$-tiled (respectively fully $p$-tiled) if there exists a $p$-tiling of $P$ (respectively there exists a $p$-tiling of $P$ and a $p$-tiling of the complementary of $P$ (i. e. the set of points of the plane which are not in the interior of $P$ )). Notice that if a polygon $P$ of $S$ can be $p$-tiled (respectively fully $p$-tiled), then for each integer $p^{\prime}$ such that $p^{\prime} \geq p, P$ can also be $p^{\prime}$-tiled (respectively fully $p^{\prime}$-tiled), since each $p$-domino can be $p^{\prime}$-tiled.

Proposition 1 Each p-polygon can be fully $p+1$-tiled.

Proof. Let $P$ be a $p$-polygon. We define the level of a $p+1$-cell, by induction, as follows : a $p+1$-cell is of level 0 if one of its corners is element of the boundary of $P$, and a $p+1$-cell is of level $i+1$ if its is not of level $j$ with $j \leq i$, and one of its corners is shared with a cell of level $i$.

We cover cells of level 0 placing tiles along the boundary, inside and outside of $P$, in such a way that no tile cuts the boundary (see figure 1). After this step is done, the non-covered part is formed from a finite set of pairwise disjoint $p$ polygons and the complementary of a big $p$-polygon which contains the previous ones.

Afterwards, cells of level 1 are covered placing tiles along the boundaries of the polygons. AS before, the non covered space after this step is still formed from a finite set of pairwise disjoint $p$-polygons and the complementary of a big $p$-polygon which contains the previous one. Thus this argument can be repeated to cover cells of level 2 , and so on for successive levels, which permits to construct a $p$-tiling of the whole plane.

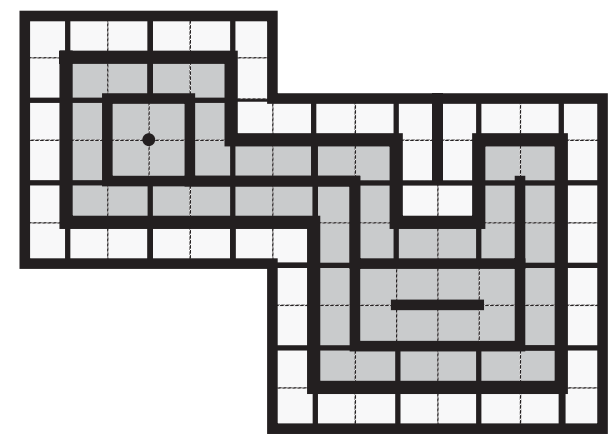

Figure 1: Construction of a $p+1$-tiling of the plane, inducing a tiling of a polygon $P$, by successive levels

Let $P$ be a $p_{0}$-polygon and $p$ be an integer such that $p>p_{0}$. Fix a $p$-tiling $T_{p, \text { comp }}$ of the complementary of $P$ (the $p$-tiling $T_{p, c o m p}$ exists from the above proposition). Using $T_{p, c o m p}$, each $p$-tiling of $P$ can be extended to the plane. 
Thus, for the following of this paper, a $p$-tiling of $P$ will be seen as a tiling of the plane.

\subsection{Height function induced by a tiling}

Proposition 2 Let $T$ be a p-tiling of a polygon $P$. The height difference of any legal cycle of $P$ for $T$ is null.

This proposition is a particular case of a more general result of J. H. Conway [2] about tilings .

Proof. (sketch) It suffices to prove it for elementary legal cycles since the height difference of each cycle is the sum of the height differences of the elementary cycles which compose it.

This is done by induction on the number of dominoes enclosed by the cycle : if only one domino is enclosed, one verifies that the proposition holds. Otherwise, the area enclosed can be cut by a legal path, which induces two new legal cycles, each of them enclosing less dominoes than the original cycle. Thus, by induction hypothesis, the height difference of both induced cycles is null, from which it is easily deduced that the height difference of the original cycle is null.

This proposition guarantees the correctness of the definition below.

Definition 1 Let $T$ be a p-tiling of a polygon $P$. The p-height function $h_{T}$ induced by $T$ is the function from the set of p-vertices which are in $P$ (possibly on the boundary), defined as follows : for each p-vertex $v, h_{T}(v)$ is the height difference of any legal path (for $T$ ) from the origin $O=(0,0)$ to $v$.

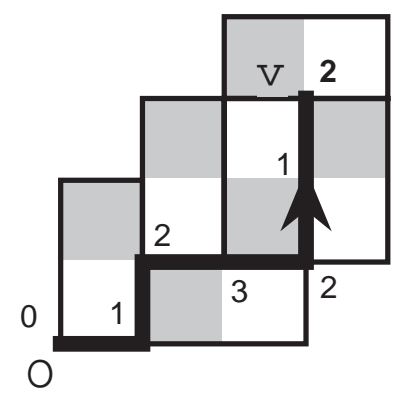

Figure 2: Computation of the height function induced by a tiling (example with $p=0)$. We obtain $h_{T}(v)=2$

Remark 1 For a fixed integer $p$, each path following the boundary of $P$ is a legal path for any $p$-tiling, thus the value of the p-height function of a p-vertex of the boundary does not depend on the p-tiling chosen.

Proposition 3 Let $\left(T, T^{\prime}\right)$ be a pair of p-tilings of a same polygon $P$. If, for each p-vertex $v$ of $P, h_{T}(v)=h_{T^{\prime}}(v)$, then $T=T^{\prime}$.

Informally, this proposition means that a height function is a way to encode a $p$-tiling. 
Proof. Let $\left(v, v^{\prime}\right)$ be a pair of neighbors $p$-vertices of $P$ such that $s p\left(v, v^{\prime}\right)=1$. We have two cases :

- the edge $\left[v, v^{\prime}\right]$ cuts no domino of $T$. Thus $h_{T}\left(v^{\prime}\right)=h_{T}(v)+1 / 2^{p}$,

- the edge joining $v$ to $v^{\prime}$ is a symmetry axis of a domino of $T$. Thus $h_{T}\left(v^{\prime}\right)=h_{T}(v)-3 / 2^{p}$ (following the boundary of this domino).

Thus, the tiling $T$ is formed from $p$-tiles whose central axis is an edge $\left[v, v^{\prime}\right]$ such that $\left|h_{T}\left(v^{\prime}\right)-h_{T}(v)\right|=3 / 2^{p}$. The same argument can be used for $T^{\prime}$, which yields : $T=T^{\prime}$.

Height functions also permit to compare some $p$-tilings. Precisely, we have the following definition :

Definition 2 (Order on $p$-tilings) Let $\left(T, T^{\prime}\right)$ be a pair of $p$-tilings of a same polygon $P$. If, for each p-vertex $v$ of $P, h_{T}(v) \leq h_{T^{\prime}}(v)$, then we say that $T \leq T^{\prime}$

\section{Limit of a sequence of height functions}

From this section, we fix an integer $p_{0}$ and a $p_{0}$-polygon $P$ of $S$.

Definition 3 Let $h$ be function defined on $V \cap P$ and $\left(h_{p}\right)_{p \geq p_{0}}$ be an infinite sequence such that, for each integer $p, h_{p}$ is the p-height function of a p-tiling of $P$. We say that $h$ is the limit of the sequence $\left(h_{p}\right)_{p>p_{0}}$ if, for each vertex $v$ of $V \cap P$ and each positive number $\epsilon$, there exists an integer $p_{1}$ such that, for each integer $p$ such that $p \geq p_{1}$, we have $\left|h_{p}(v)-h(v)\right|<\epsilon$.

The goal of this paper is to give a characterization of those limits functions. We first give two necessary conditions satisfied by each limit function.

\subsection{The boundary condition}

Proposition 4 Let $T$ be a p-tiling of $P$, with $p>p_{0}$, and $h_{p}$ denote its height function. For each p-vertex $v$ of the boundary of $P$, we have $\left|h_{p}(v)\right| \leq 1 / 2^{p}$.

Proof. Let $\left(O=v_{0}, v_{1}, v_{2}, \ldots, v_{n}=O\right)$ denote the $p$-cycle following the boundary of $P$, counterclockwise. We claim that, for each integer $i$ such that $0 \leq i \leq n / 2$, we have $h_{p}\left(v_{2 i}\right)=0$. This is obvious by induction on $i$, once it has be remarked that the length of the line segment $\left[v_{2 i}, v_{2 i+2}\right]$ is included in the boundary of $P$ of and moreover, the length of $\left[v_{2 i}, v_{2 i+2}\right]$ is $2 / 2^{p}$.

On the other hand, for each integer $i$ such that $0 \leq i<n / 2$, we have : $\left|h_{p}\left(v_{2 i+1}\right)-h_{p}\left(v_{2 i}\right)\right| \leq 1 / 2^{p}$, which concludes the proof.

Corollary 1 (boundary condition) If $h$ is the limit of a sequence of tiling functions, then for each vertex $v$ of $S$ which is on the boundary of $P$, we have $h(v)=0$.

Proof. obvious. 


\subsection{The Lipschitz condition}

Definition 4 Let $\left(v, v^{\prime}\right)$ be a pair of distinct p-points. A p-path $(v=$ $\left.v_{0}, v_{1}, \ldots, v_{n}=v^{\prime}\right)$ is called increasing (respectively decreasing) if, for each integer $i$ such that $0 \leq i<n, s p\left(v_{i}, v_{i+1}\right)=1$ (respectively $\left.s p\left(v_{i}, v_{i+1}\right)=-1\right)$.

We define $\delta_{p}\left(v, v^{\prime}\right)$ as the length of a shortest increasing path from $v$ to $v^{\prime}$

Lemma 1 Let $v=\left(a / 2^{p}, b / 2^{p}\right)$ and $v^{\prime}=\left(a^{\prime} / 2^{p}, b^{\prime} / 2^{p}\right)$ be p-points. We have the double inequality :

$$
2 d_{\infty}\left(v, v^{\prime}\right)-1 / 2^{p} \leq \delta_{p}\left(v, v^{\prime}\right) \leq 2 d_{\infty}\left(v, v^{\prime}\right)+1 / 2^{p}
$$

where $d_{\infty}\left(v, v^{\prime}\right)$ denotes the value $\max \left(\left|a-a^{\prime}\right|,\left|b-b^{\prime}\right|\right) / 2^{p}$.

Proof. By symmetry, we can assume without loss of generality that $a \leq a^{\prime}$, $b \leq b^{\prime}$, and $b^{\prime}-b \leq a^{\prime}-a$.

Each increasing path from $v$ to $v^{\prime}$ contains at least (a'- a) rightwards $p$ moves, and two rightwards $p$-moves are necessarily separated by at least one vertical $p$-move. This gives the first inequality.

The second inequality is obtained constructing an increasing path from $v^{\prime}$ to $v$ of length at most $2 d_{\infty}\left(v, v^{\prime}\right)$. Let $v^{\prime \prime}=\left(\left(a+b^{\prime}-b\right) / 2^{p}, b^{\prime} / 2^{p}\right)$. Since $v^{\prime}$ and $v$ " have the same second coordinate, there obviously exists an increasing path, from $v$ " to $v^{\prime}$, formed from at most $2\left(a^{\prime}-a+b-b^{\prime}\right)+1 p$-moves.

Moreover, since $v "$ and $v$ are on a same straight line which is parallel to the diagonal line (whose equation is : $x=y$ ), there exists an increasing path from $v$ " to $v$ formed from $2\left(b^{\prime}-b\right) p$-moves.

Thus, concatening the previous paths, we obtain an increasing path from $v^{\prime}$ to $v$ formed from at most $2\left(a^{\prime}-a\right)+1$ paths, which concludes the proof.

Proposition 5 Let $T$ be a p-tiling of $P$, with $p>p_{0}$, and $h_{p}$ denote its height function. For each pair $\left(v, v^{\prime}\right)$ of vertices of $V_{p} \cap P$, we have the inequality :

$$
h_{p}\left(v^{\prime}\right)-h_{p}(v) \leq \delta_{p}\left(v, v^{\prime}\right)
$$

Proof. Let $\left(v=v_{0}, v_{1}, \ldots, v_{n}=v\right)$ be an increasing path from $v$ to $v^{\prime}$. We have :

$$
h_{p}\left(v^{\prime}\right)-h_{p}(v)=\sum_{i=0}^{n-1}\left(h_{p}\left(v_{i+1}\right)-h_{p}\left(v_{i}\right)\right)
$$

But, for each integer $i$ such that $0 \leq i<n$, we have : $h_{p}\left(v_{i+1}\right)-h_{p}\left(v_{i}\right) \leq 1 / 2^{p}$, which yields : $h_{p}\left(v^{\prime}\right)-h_{p}(v) \leq n / 2^{p}$.

Corollary 2 (Lipschitz condition) If $h$ is the limit of a sequence of tiling functions, then for each pair $\left(v, v^{\prime}\right)$ of vertices of $V \cap P$, we have the inequality

$$
\left|h(v)-h\left(v^{\prime}\right)\right| \leq 2 d_{\infty}\left(v, v^{\prime}\right)
$$

Proof. From the previous inequalities, for each integer $p$, we have :

$$
\left|h_{p}(v)-h_{p}\left(v^{\prime}\right)\right| \leq \max \left(\delta_{p}\left(v, v^{\prime}\right), \delta_{p}\left(v^{\prime}, v\right)\right) \leq 2 d_{\infty}\left(v, v^{\prime}\right)+1 / 2^{p}
$$

which obviously gives the result. 
Each function $h$ simultaneously satisfying the boundary condition and the Lipschitz condition can be extended by continuity from $V \cap P$ to $P$, since $V \cap P$ is a dense subset of $P$ and the Lipschitz condition implies the uniform continuity.

Moreover, the function $h$ can be extended to the complementary of $P$, stating $h(v)=0$ for each point $v$ outside of $P$.

The Lipschitz condition is actually be transformed into a local condition. This fact is formally expressed by the following proposition.

Proposition 6 Let $h$ be a real function, defined on the plane, such that $h$ is null outside of $P$ and on the boundary of $P$.

The function $h$ is the extensions of a function which satisfies the Lipschitz condition if and only if $h$ satisfies the local following condition:

for each point $v$ of $P$, there exists a positive real $\epsilon$ such that, for each point $v^{\prime}$ of $P$ such that $d_{\infty}\left(v, v^{\prime}\right)<\epsilon$, we have : $\left|h(v)-h\left(v^{\prime}\right)\right| \leq 2 d_{\infty}\left(v, v^{\prime}\right)$.

Proof. The direct part of this proposition is obvious. Conversely, assume that $h$ satisfies the local condition of the proposition, and let $\left(v, v^{\prime}\right)$ be a pair of elements of $V \cap P$. Consider the set

$$
\left\{v^{\prime \prime} \in\left[v, v^{\prime}\right] \text { such that for each point } w \text { of }\left[v, v^{\prime \prime}\right],|h(v)-h(w)| \leq 2 d_{\infty}(v, w)\right\}
$$

This set is closed since $h$ is continuous, and this set is open from the local condition. Moreover this set is not empty, thus, since the segment $\left[v, v^{\prime}\right]$ is a connected topological set, the set above necessarily equals $\left[v, v^{\prime}\right]$, which gives the Lipschitz condition.

\section{Construction of a $p$-approximation}

Definition 5 Let $h$ be a function defined on $V_{p} \cap P$. A tiling p-approximation of $h$ is a p-height function $h_{p}$ is a tiling p-approximation of $h$, of parameter $\alpha$ if, for each p-vertex $v$ of $P$, we have : $\left|h(v)-h_{p}(v)\right| \leq \alpha$

Let $h$ be a function defined on $V \cap P$. Assume that, for each integer $p$ such that $p>p_{0}, h$ has a $p$-approximation $h_{p}$ of parameter $\alpha_{p}$, and the sequence $\left(\alpha_{p}\right)_{p>p_{0}}$ converges to 0 , then $h$ is the limit of the sequence $\left(h_{p}\right)_{p>p_{0}}$.

In this section we will prove the theorem below.

Theorem 1 Let $h$ be a function defined on $V \cap P$, simultaneously satisfying the boundary condition and the Lipschitz condition.

For each each integer $p$ such that $p>p_{0}$, there exists a tiling $p$-approximation of $h$, of parameter $3 / 2^{p}$.

This theorem has the following corollary, since the limit the sequence from the above $p$-approximations of $h$ is obviously $h$ :

Corollary 3 (Characterization of limit functions) A function $h$, defined on $V \cap P$ is the limit of a sequence of tiling functions if and only if h simultaneously satisfies the boundary condition and the Lipschitz condition.

The proof of the theorem needs to extend the notion of height function, and to point out algebraic properties of these functions. We do it in the the following parts. 


\subsection{Extension of the notion of height function}

Proposition 7 Let $h_{p}$ be a function from the set of $p$-vertices in $P$ to the set of real numbers such that:

- $h_{p}(O)=0$,

- for each pair $\left(v, v^{\prime}\right)$ of neighbors p-vertices such that $s p\left(v, v^{\prime}\right)=1$, either $h_{p}\left(v^{\prime}\right)=h_{p}(v)+1 / 2^{p}$ or $h_{p}\left(v^{\prime}\right)=h_{p}(v)-3 / 2^{p}$,

- if, moreover, the edge $\left[v, v^{\prime}\right]$ is on the boundary of $P$, then $h_{p}\left(v^{\prime}\right)=h_{p}(v)+$ $1 / 2^{p}$.

There exists a p-tiling $T$ of $P$ such that $h_{p}=h_{T}$.

Proof. Let $\left(v_{0}, v_{1}, v_{2}, v_{3}, v_{4}=v_{0}\right)$ be a cycle around a white $p$-cell, counterclockwise. The second constraint of the proposition implies that we have three vertices $w_{i}$ such that $h\left(v_{i+1}\right)=h\left(v_{i}\right)+1$ and a unique vertex $v_{j}$ such that $h\left(v_{j+1}\right)=h\left(v_{j}\right)-3$. One easily obtains a symmetric condition for black $p$-cells.

Thus, the set $T$ of $p$-dominoes which are cut into both halves by an edge, say $\left[v, v^{\prime}\right]$, such that $\left|h(v)-h\left(v^{\prime}\right)\right|=3$, is a $p$-tiling of $P$. One obviously verifies, (by induction on the length of a shortest legal path from $O$ to $v$ ) that, for each $p$-vertex $v$ of $P, h(v)=h_{T}(v)$.

Definition 6 The 4-congruence of a p-vertex $v=\left(a / 2^{p}, b / 2^{p}\right)$ (denoted by cong $\left.g_{p}(v)\right)$, is the value of $Z / 4 Z$ given below :

- if $(a, b)=(0,0)[2]$, then $\operatorname{cong}_{p}(v)=0[4]$,

- if $(a, b)=(1,0)[2]$, then $\operatorname{cong}_{p}(v)=1[4]$,

- if $(a, b)=(1,1)[2]$, then $\operatorname{cong}_{p}(v)=2[4]$,

- if $(a, b)=(0,1)[2]$, then $\operatorname{cong}_{p}(v)=3[4]$.

Proposition 8 For any p-tiling $T$ and each p-vertex $v$ of $P$, we have : $2^{p} h_{T}(v)=\operatorname{cong}_{p}(v)[4]$.

Proof. obvious by induction on the length of a shortest path (staying in $P$ ) from $O$ to $v$.

We want to keep the above properties of height function of tilings, except the boundary constraint (in order to construct tilings of the whole plane). This is done by the definition below :

Definition 7 A generalized $p$-height function $h_{p}$ is a function from the set $V_{p}$ of $p$-vertices in $P$ to the set of real numbers such that:

- for each pair $\left(v, v^{\prime}\right)$ of neighbors p-vertices such that $\operatorname{sp}\left(v, v^{\prime}\right)=1$, either $h_{p}\left(v^{\prime}\right)=h_{p}(v)+1 / 2^{p}$ or $h_{p}\left(v^{\prime}\right)=h_{p}(v)-3 / 2^{p}$,

- for each vertex $v$ of $V_{p}, 2^{p} h_{p}(v)=\operatorname{cong}_{p}(v)[4]$. 
Proposition 9 (Fundamental example) Some generalized p-height functions can be constructed as follows : take a vertex $v_{0}$ and $z$ a value such that $2^{p} z=\operatorname{cong}_{p}\left(v_{0}\right)[4]$.

The function $h_{p, v_{0}, z}$, defined by $: h_{p, v_{0}, z}(v)=z+\delta_{p}\left(v_{0}, v\right)$, is a generalized p-height function.

The function $h_{p, v_{0}, z}^{\prime}$, defined by $: h_{p, v_{0}, z}^{\prime}(v)=z-\delta_{p}\left(v, v_{0}\right)$, is a generalized p-height function.

Proof. The second condition $\left(h_{p, v_{0}, z}(v)=\operatorname{cong}_{p}(v)[4]\right)$ is obvious, by induction on $\delta_{p}\left(v_{0}, v\right)$.

Moreover, for each pair $\left(v, v^{\prime}\right)$ of neighbors $p$-vertices such that $s p\left(v, v^{\prime}\right)=1$, we have :

$$
\delta_{p}\left(v_{0}, v^{\prime}\right) \leq \delta_{p}\left(v_{0}, v\right)+1 / 2^{p}
$$

using an increasing path from $v_{0}$ to $v^{\prime}$ passing through $v$, starting by a shortest path from $v_{0}$ to $v$.

On the other hand, using a similar method, we obtain

$$
\delta_{p}\left(v_{0}, v\right) \leq \delta_{p}\left(v_{0}, v^{\prime}\right)+3 / 2^{p}
$$

Those equalities yield that :

$$
h_{p, v_{0}, z}(v)-3 / 2^{p} \leq h_{p, v_{0}, z}\left(v^{\prime}\right) \leq h_{p, v_{0}, z}(v)+1 / 2^{p}
$$

which give the first condition of the previous definition : among the five possible values of $h_{p, v_{0}, z}\left(v^{\prime}\right)$ left by the previous double inequality, only the two extremal ones are possible, because of the congruence condition.

The function $h_{p, v_{0}, z}^{\prime}$ is treated in a similar way.

\subsection{Lattice properties}

Proposition 10 Let $\left(h_{p}, h_{p}^{\prime}\right)$ be a pair of generalizedp-height function. The functions $h_{p, \min }$ and $h_{p, \max }$, defined by $h_{p, \min }(v)=\min \left(h_{p}(v), h_{p}^{\prime}(v)\right)$ and $h_{p, \max }(v)=\max \left(h_{p}(v), h_{p}^{\prime}(v)\right)$, are generalized $p$-height functions.

Proof. We prove this lemma for $h_{p, \min }$ (the proof for $h_{p, \max }$ is similar) using proposition 7 . The second constraint is obviously satisfied.

Let $\left(v, v^{\prime}\right)$ be a pair of neighbors $p$-vertices of $P$ such that $s p\left(v, v^{\prime}\right)=1$. Assume that $h_{p}(v)<h_{p}^{\prime}(v)$. Then, from proposition 8, we have : $h_{p}(v) \leq$ $h_{p}^{\prime}(v)-4 / 2^{p}$. On the other hand, $h_{p}\left(v^{\prime}\right) \leq h_{p}(v)+1 / 2^{p}$ and $h_{p}^{\prime}\left(v^{\prime}\right) \geq h_{p}^{\prime}(v)-$ $3 / 2^{p}$. Thus :

$h_{p}\left(v^{\prime}\right) \leq h_{p}(v)+1 / 2^{p} \leq h_{p}^{\prime}(v)-4 / 2^{p}+1 / 2^{p} \leq h_{p}^{\prime}\left(v^{\prime}\right)+3 / 2^{p}-4 / 2^{p}+1 / 2^{p}=h_{p}^{\prime}\left(v^{\prime}\right)$

This proves that if $h_{p}(v)<h_{p}^{\prime}(v)$ then $h_{p, \min }\left(v^{\prime}\right)=h_{p}\left(v^{\prime}\right)$. Consequently (since, of course, $\left.h_{p, \min }(v)=h_{p}(v)\right) h_{p, \min }\left(v^{\prime}\right)-h_{p, \min }(v)=h_{p}\left(v^{\prime}\right)-h_{p}(v)$, which guarantees the first constraint of proposition 7 .

The case when $h_{p}(v)>h_{p}^{\prime}(v)$ is treated with the same kind of argument and the case when $h_{p}(v)=h_{p}^{\prime}(v)$ is obvious.

Corollary 4 The set of p-tilings of $P$, ordered as defined in section 2 , is a distributive lattice. 
Proof. obvious.

This lattice structure has been previously studied with more details in [6]

Corollary 5 Let $v_{0}$ be a p-vertex and $z$ be a value such that $2^{p} z=\operatorname{cong}_{p}\left(v_{0}\right)[4]$. The set $H_{p, v_{0}, z}$, formed from generalized p-height functions $h_{p}$ such that $h_{p}\left(v_{0}\right)=z$, is a distributive lattice (for the canonical order on real functions).

Moreover, the function $h_{p, v_{0}, z}$ is the maximum of $H_{p, v_{0}, z}$ and $h_{p, v_{0}, z}^{\prime}$ is minimum of $H_{p, v_{0}, z}$

Proof. The first part of this proposition is obvious from proposition 10. The second part is a direct consequence of proposition 5 .

\subsection{Applications}

We now have the necessary tools to construct a $p$-approximation. This will be done below using the following proposition.

Proposition 11 Let $h_{p, P_{\max }}$ denote the height function of the maximum $p$ tiling of $P$, and $h_{p, P_{\text {min }}}$ denote the height function of the minimum p-tiling of $P$. For each vertex $v$ of $V_{p} \cap P$, we have the equalities :

$$
\begin{aligned}
& h_{p, P_{\max }}(v)=\min _{v^{\prime} \in \delta P}\left(h_{p, v^{\prime}, h_{p}\left(v^{\prime}\right)}(v)\right) \\
& h_{p, P_{\min }}(v)=\max _{v^{\prime} \in \delta P}\left(h_{p, v^{\prime}, h_{p}\left(v^{\prime}\right)}^{\prime}(v)\right)
\end{aligned}
$$

where $\delta P$ denotes the boundary of $P$, and $h_{p}\left(v^{\prime}\right)$ is the common value of all the p-height functions in $v^{\prime}$.

Proof. For each $p$-vertex $v^{\prime}$ of $\delta P, h_{p, P_{\max }}$ can be seen as an element of $H_{p, v^{\prime}, h_{p}\left(v^{\prime}\right)}$, which yields that $h_{p, P_{\max }} \leq h_{p, v^{\prime}, h_{p}\left(v^{\prime}\right)}$. Thus for each vertex $v$ of $V_{p} \cap P$, we have

$$
h_{p, P_{\max }}(v) \leq \min _{v^{\prime} \in \delta P}\left(h_{p, v^{\prime}, h_{p}\left(v^{\prime}\right)}(v)\right)
$$

On the other hand, $\min _{v^{\prime} \in \delta P}\left(h_{p, v^{\prime}, h_{p}\left(v^{\prime}\right)}\right.$ induces a tiling of $P$; which gives the converse inequality. The function $h_{p, P_{\min }}$ is treated in a similar way.

Corollary 6 Let $h$ be a function, defined on $V \cap P$, such that $h$ simultaneously satisfies the boundary condition and the Lipschitz condition. For each vertex $v$ of $V_{p} \cap P$, we have :

$$
h_{p, P_{\min }}(v)-2 / 2^{p} \leq h(v) \leq h_{p, P_{\max }}(v)+2 / 2^{p}
$$

This corollary is an application of lattice structures to limits of tilings.

Proof. From proposition 11, there exists a vertex $v^{\prime}$ of $V_{p} \cap \delta P$ such that $h_{p, P_{\max }}(v)=h_{p}\left(v^{\prime}\right)+\delta_{p}\left(v^{\prime}, v\right)$. On the other hand, we recall that

$$
h(v)-h\left(v^{\prime}\right) \leq 2_{\infty}\left(v, v^{\prime}\right) \leq \delta_{p}\left(v^{\prime}, v\right)+1 / 2^{p}
$$

Thus, since $h\left(v^{\prime}\right)=0$, we obtain :

$$
h(v) \leq h_{p, P_{\max }}(v)-h_{p}\left(v^{\prime}\right)+1 / 2^{p} \leq h_{p, P_{\max }}(v)+1 / 2^{p}+1 / 2^{p}
$$

which is the second inequality of the proposition. The first inequality is obtained in a symmetric way. 
Definition 8 For each function $h$, defined on $V_{p} \cap P$, we define the function round $_{p, h}$ by : for each vertex $v$ of $V_{p} \cap P, \operatorname{round}_{p, h}(v)=a_{v} / 2^{p}$ where $a_{v}$ denotes the unique integer such that $2^{p} a_{v}=\operatorname{cong}(v)[4]$ and $\left(a_{v}-2\right) / 2^{p} \leq h(v)<\left(a_{v}+\right.$ $2) / 2^{p}$ (except in the extremal particular case when $h(v)=h_{p, P_{\max }}(v)+2 / 2^{p}$; in this case, we state round r,$\left.h(v)=h_{p, P_{\text {max }}}(v)\right)$.

We also define the function approx ${ }_{p, h}$ by : for each vertex $v$ of $V_{p} \cap P$,

$$
\operatorname{approx}_{p, h}(v)=\min _{v^{\prime} \in P}\left(h_{p, v^{\prime}, \operatorname{round}_{p, h}\left(v^{\prime}\right)}(v)\right)
$$

Remark 2 Notice that, from the above proposition, for each vertex $v$ of $V_{p} \cap P$, we have

$$
h_{p, P_{\text {min }}}(v) \leq \operatorname{round}_{p, h}(v) \leq h_{p, P_{\max }}(v)
$$

Especially, if $v$ is on the boundary of $P, \operatorname{round}_{p, h}(v)$ is the common value of all p-tilings height functions.

Proposition 12 The function approx $_{p, h}$ is the tiling function of a p-tiling of $P$.

Proof. We will prove that there exists a $p$-tiling of $P$, for each $p$-vertex $v$ of the boundary of $P$, $\operatorname{approx}_{p, h}(v)=h_{p, P_{\text {min }}}(v)$. This fact gives the result from proposition 7 .

First notice that :

$$
\operatorname{approx}_{p, h}(v) \leq h_{p, v, \operatorname{round}_{p, h}(v)}(v)=\operatorname{round}_{p, h}(v)
$$

and $\operatorname{round}_{p, h}(v)=h_{p, P_{\text {min }}}(v)$ from remark 2. Thus

$$
\operatorname{approx}_{p, h}(v) \leq h_{p, P_{m i n}}(v)
$$

Moreover, for each vertex $v^{\prime}$ of $V_{p} \cap P$, we have the inequalities

$$
h_{p, P_{\text {min }}}(v)-h_{p, P_{\text {min }}}\left(v^{\prime}\right) \leq \delta\left(v^{\prime}, v\right)
$$

from proposition 5 , and

$$
h_{p, P_{\text {min }}}\left(v^{\prime}\right) \leq \operatorname{round}_{p, h}\left(v^{\prime}\right)
$$

from remark 2. Adding these inequalities, we obtain

$$
h_{p, P_{\text {min }}}(v) \leq h_{p, v^{\prime}, \operatorname{round}_{p, h}\left(v^{\prime}\right)}(v)
$$

which yields that

$$
h_{p, P_{\min }}(v) \leq \operatorname{approx}_{p, h}(v)
$$

and finishes the proof.

Proposition 13 For each vertex $v$ of $V_{p} \cap P$, we have

$$
\left|h(v)-\operatorname{approx}_{p, h}(v)\right| \leq 3 / 2^{p}
$$


Proof. Let $v^{\prime}$ be any element of $V_{p} \cap P$. for each vertex $v$ of $V_{p} \cap P$, we have :

$$
h(v)-h\left(v^{\prime}\right) \leq 2 d_{\infty}\left(v^{\prime}, v\right) \leq \operatorname{dir}\left(v^{\prime}, v\right)+1 / 2^{p}
$$

Thus

$h(v) \leq h\left(v^{\prime}\right)+\operatorname{dir}\left(v^{\prime}, v\right)+1 / 2^{p} \leq \operatorname{round}_{p, h}\left(v^{\prime}\right)+2 / 2^{p}+\operatorname{dir}\left(v^{\prime}, v\right)+1 / 2^{p}=h_{p, v^{\prime}, \operatorname{round}_{p, h}\left(v^{\prime}\right)}(v)+3 / 2^{p}$

which gives : $h(v) \leq \operatorname{approx}_{p, h}(v)+3 / 2^{p}$.

On the other hand,

$$
h(v) \geq \operatorname{round}_{p, h}(v)-2 / 2^{p}=h_{p, v, \operatorname{round}_{p, h}(v)}(v)-2 / 2^{p}
$$

which yields : $h(v) \geq \operatorname{approx}_{p, h}(v)-2 / 2^{p}$.

This last proposition finishes the proof of the theorem claimed at the beginning of this section.

\section{$5 \quad$ Extremal examples}

We finish this paper giving an example of non-trivial limit function, which is the highest one.

Proposition 14 Let $h_{\max }$ be the function defined by:

$$
h_{\max }(v)=2 d_{\infty}(v, \delta P) \quad\left(i . e .2 \min _{v^{\prime \prime} \in \delta P}\left(d_{\infty}\left(v, v^{\prime \prime}\right)\right)\right)
$$

The function $h_{p, P_{\max }}$ is an approximation of $h_{\max }$, with parameter $2 / 2^{p}$.

Proof. Let $v$ be any $p$-point. For each $p$-vertex $v$ " of the boundary of $p$, we have, from lemma 1 :

$$
h(v) \leq 2 d_{\infty}\left(v^{\prime \prime}, v\right) \leq \delta\left(v^{\prime \prime}, v\right)+1 / 2^{p}
$$

Thus, from proposition 4 :

$$
h(v) \leq h_{p, v^{\prime \prime}, h_{p}\left(v^{\prime \prime}\right)}(v)+2 / 2^{p}
$$

where $h_{p}(v ")$ denotes the only possible value for a tiling height function in $v$ ". Thus, from proposition 11 :

$$
h(v) \leq h_{p, P_{\max }}(v)+2 / 2^{p}
$$

On the other hand notice that, since the boundary of $P$ is compact, there exists a point $v^{\prime}$ of the boundary of $P$, such that $d_{\infty}(v, \delta P)=d_{\infty}\left(v, v^{\prime}\right)$. Moreover, one easily sees that $v^{\prime}$ can be chosen in such a way that $v^{\prime}$ is a corner of $P$, and, consequently, a $p$-vertex.

From lemma 1, we have

$$
h(v)=2 d_{\infty}\left(v, v^{\prime}\right) \geq \delta\left(v^{\prime}, v\right)-1 / 2^{p}
$$

which yields :

$$
h(v) \geq h_{p, v^{\prime}, h_{p}\left(v^{\prime}\right)}(v)-2 / 2^{p}
$$

Thus

$$
h(v) \geq h_{p, P_{\max }}(v)-2 / 2^{p}
$$

which concludes the proof. 

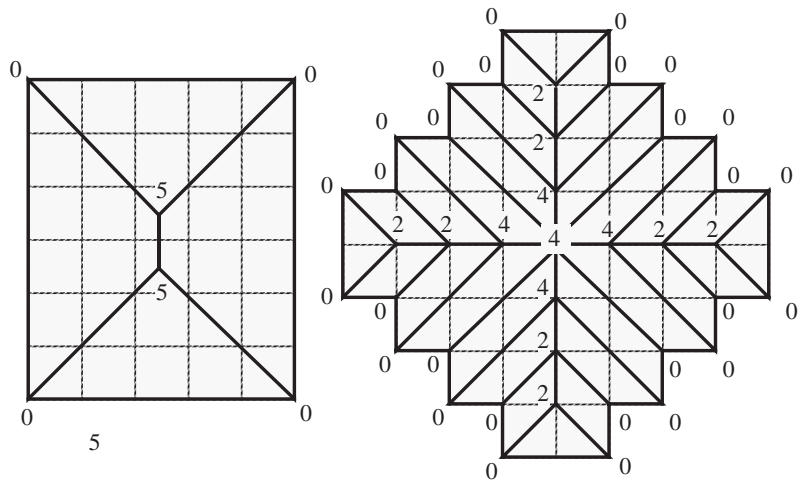

Figure 3: Construction of $h_{\max }$ for a rectangle and an Aztec diamond. The polygon is divided into polygonal regions on which $h_{m} a x$ is linear. The numbers indicate the values of $h_{\max }$ in the corners of the regions

\section{References}

[1] R. Berger, The indecidability of the domino problem, Memoirs of the American Mathematical Society 66 (1966)

[2] J. H. Conway, J. C. Lagarias, Tiling with Polyominoes and Combinatorial Group Theory, Journal of Combinatorial Theory, A 53, (1990) 183-208 .

[3] C. Kenyon, R. Kenyon, Tiling a polygon with rectangles, Proceedings of $33^{\text {rd }}$ FOCS (1992) 610-619.

[4] J. C. Lagarias, D. S. Romano, A Polyomino Tiling of Thurston and its Configurational Entropy, Journal of Combinatorial Theory, A 63, (1993) 338-358.

[5] J. G. Propp, Lattice structure for orientations of graphs, preprint., (1993).

[6] E. Rémila, On the lattice structure of the set of tilings of a simply connected figure with dominoes, proceedings of the $3^{\text {rd }}$ International Conference on Orders, Algorithms and Applications (ORDAL) LNCS Springer (to appear) 12 pages.

[7] E. Rémila, Tiling groups : new applications in the triangular lattice, Discrete and Computational Geometry (1998) 20, p 189-204.

[8] W. P. Thurston, Conway's tiling group, American Mathematical Monthly, (1990) $757-773$. 